\title{
Linking chromatin dynamics, cell fate plasticity, and tissue homeostasis in adult mouse hair follicle stem cells
}

\author{
Jayhun Lee ${ }^{1,2}$ and Tudorita Tumbar ${ }^{1 *}$ \\ ${ }^{1}$ Department of Molecular Biology and Genetics, Cornell University, Ithaca, NY 14853, USA \\ ${ }^{2}$ Present address: Morgridge Institute for Research, University of Wisconsin-Madison, Madison, Wisconsin 53715, USA \\ (Received 4 March, 2017; accepted 10 May, 2017)
}

Cellular plasticity for fate acquisition is associated with distinct chromatin states, which include histone modifications, dynamic association of chromatin factors with the DNA, and global chromatin compaction and nuclear organization. While embryonic stem cell (ESC) plasticity in vitro and its link with chromatin states have been characterized in depth, little is known about tissue stem cell plasticity in vivo, during adult tissue homeostasis. Recently, we reported a distinct globally low level of histone $\mathrm{H} 3 \mathrm{~K}$ 4/9/27me3 in mouse hair follicle stem cells (HFSCs) during quiescence. This occurred at the stage preceding fate acquisition, when HFSC fate plasticity must be at its highest. This hypomethylated state was required for proper skin homeostasis and timely hair cycle. Here, we show both in the live tissue and in cell culture that at quiescence HFSCs have higher exchange rates for core histone $\mathrm{H} 2 \mathrm{~B}$ when compared with proliferative or differentiated cells. This denoted a hyperdynamic chromatin state, which was previously associated with high cell fate plasticity in ESCs. Moreover, we find that quiescent HFSCs display a higher propensity for dedifferentiation in response to Yamanaka's reprogramming factors in vivo. These results further support our recent model in which HFSCs render their chromatin into a specific state at quiescence, which is attuned to higher cell fate plasticity.

\section{MAIN TEXT}

Plasticity has been previously linked with specialized chromatin states in pluripotent stem cells. For example, rapid exchange of chromatin bound factors, referred to as hyperdynamic chromatin, is required for embryonic stem cells (ESCs) to differentiate $(1,2)$. However, most of such studies were performed under in vitro culture conditions that do not faithfully recapitulate in vivo settings. Many (though not all) adult tissue stem cells reside in G0 quiescence (do not divide) in their niche for long time periods. Tissue stem cell fate is determined by asymmetric cell division in some tissues and by environmental cues or stochastic events in others $(3,4)$. Understanding the link between chromatin states and plasticity of tissue stem cells is poorly understood, but is limited by their scarcity and accessibility in many cases. Mouse hair follicles harbor stem cells (HFSCs) that undergo cyclic phases of proliferation and quiescence, termed hair cycle (Fig. 1A). The hair cycle is synchronized across the mouse skin, offering an easy access and high yield for isolation and imaging of stem cells at distinct stages of proliferation and quiescence, making it an excellent adult tissue model for understanding the biology of tissue stem cells and the link between chromatin features and plasticity.

Adult HFSCs positioning relative to the niche (bulge) occurs during catagen, the regression phase of the hair cycle, and dictates the ultimate stem cell fate to either differentiate or self-renew $(5,6)$ (Fig. 1A). Stem cells positioned below the bulge in the hair germ $(\mathrm{Hg})$ proliferate rapidly and differentiate without self-renewal to matrix progenitor cells at anagen (growth phase) onset, which further give rise to the terminally differentiated lineages on the hair follicle, including the inner root sheath and the hair shaft $(7,8)$. Subsequently, early anagen bulge stem cells replenish their own pool by symmetric stem cell population expansion within the bulge, without further cellular contribution to the differentiated compartment until the next catagen (5). Therefore,

${ }^{*}$ Corresponding author: Tudorita (Doina) Tumbar, Ph.D., Associate Professor

258 Biotechnology Building, Department of Molecular Biology and Genetics

Cornell University, Ithaca, NY 14853

E-mail: tt252@cornell.edu

Fax: 607-255-6249; Phone: Office 607-255-6542; Lab 607-255-0518 
HFSCs most naive cell fate state is at catagen, during the stem cell positioning phase. Intriguingly, we have recently shown that bulge cells in catagen display distinct genomewide hypomethylation of several histone modifications (H3 K4/9/27me3) (9). Hypomethylation of H3 K9/27me3 has been previously associated with increased cell fate plasticity in ESCs and in quiescent T-cells in vitro $(10,11)$. We found that this specialized chromatin state accompanies differential regulation of multiple histone methylases and demethylases, and is associated with BMP signaling (9), previously shown to be critical for proper maintenance of quiescent state (12). Moreover, blocking the establishment of hypomethylation at catagen via chemical inhibition of $\mathrm{H} 3 \mathrm{~K} 4 / 9 / 27$ methylases led to a pronounced delay in anagen onset. These findings demonstrate that the same signals (BMP) that induce quiescence in a tissue stem cell at the stage of their cell fate decision, also promote a plasticity-associated chromatinstate (histone hypomethylation), which is essential for subsequent behavior of tissue stem cells and for normal tissue regeneration. These findings led us to further investigate another chromatin state previously associated with plasticity in ESCs in vitro, namely chromatin factor exchange rates, and see how they apply to HFSCs during hair cycle. Moreover, we asked whether in their most naïve cell fate state, during quiescence, HFSCs are also more prone to de-differentiation in response to reprogramming factors, which is another measure of cell plasticity.

In order to examine the differential chromatin states of HFSCs, we first assayed chromatin factor exchange rates via fluorescence recovery after photobleaching (FRAP). Chromatin factor exchange has been previously associated with plasticity in ESCs, and was shown to be required for ESCs to differentiate (1). First, we examined chromatin factor exchange rates in HFSCs by live imaging of skin explants, within 30 minutes after tail excision from transgenic mice bearing K5-tTA (Keratin 5 promoter-driven tet-repressible transactivator) and $p$ TRE-H2BGFP (Tet-responsive regulatory element-driven GFP-fused histone $\mathrm{h} 2 \mathrm{~b}$ ), expressing high level of H2B-GFP throughout the skin epithelia without doxycycline treatment (13) (Figures 1B and 1C).

We performed FRAP on cells from hair follicle that based on morphology were at distinct hair cycle stages: (1) anagen, in proliferative HFSCs and (2) late catagen/ early telogen, in quiescent HFSCs (Fig. 1C). Strikingly, quiescent (q) HFSCs identified by the bulge location at late catagen/telogen displayed faster and greater H2B-GFP recovery rates than proliferative (p) bulge HFSCs at anagen. More differentiated hair follicle cells located in the matrix and inner layers (Mx/IL) showed the lowest rates of recovery (Figures 1D-1F). These data suggest that quiescent HFSCs localized in their in vivo niche display hyperdynamic chromatin as defined by H2B-GFP high exchange rates, in line with their potential increased plasticity at this stage.

Next, we asked if the H2B-GFP exchange rates are directly correlated with intrinsic states of stem cell quiescence versus proliferation per se, or whether they were related with distinct skin environmental cues at different hair cycle stages. For this we used skin-derived keratinocytes from transgenic mice expressing K14-rtTA (Keratin 14 promoter-driven tet-inducible transactivator) (14) and pTRE-H2BGFP (13), cultured in vitro and re-assessed the correlation between quiescence and hyperdnamic chromatin state (Fig. 2A). We induced quiescence, proliferation, and differentiation by subjecting our cells to low serum, low Calcium, and high Calcium, respectively, while treating cells with doxycycline for at least 48 hours. Interestingly, our results in vitro were similar to those obtained in vivo, as quiescent keratinocytes display the highest H2B-GFP exchange rates, when compared to proliferating and differentiated keratinocytes (Figures 2A-2C). These rates were not influenced by differences in protein abundance, since the overall H2B-GFP protein expression levels (measured by total GFP fluorescence level in each cell) were mostly comparable between conditions (Figures 2D and 2E). Intriguingly, these data are in line with the data from cultured ESCs (1) where ESCs, compared to more lineage-restricted cell types, showed the highest exchange rates of histone proteins. Moreover, these data agree with our previous report, in which quiescent HFSCs have been shown to have globally reduced histone epigenetic marks (9), another chromatin feature associated with higher plasticity in ESCs and T cell progenitors $(10,11)$.

Finally, we asked if quiescent HFSCs may show increased propensity for de-differentiation when compared to proliferative HFSCs in vivo. Higher de-differentiation ability of in vitro cultured quiescent versus proliferative $\mathrm{T}$ cell progenitors was previously reported in nuclear transfer assays, and this was dependent on the hypomethylation state of quiescent cells (11). In order to assess the HFSCs propensity to de-differentiate, we examined their gene expression response to the over-expression of Yamanaka's reprograming factors (4F) in vivo (15). Previous studies have shown that terminally differentiated cell types undergo a series of de-differentiation steps before turning into fully reprogrammed cells (16).

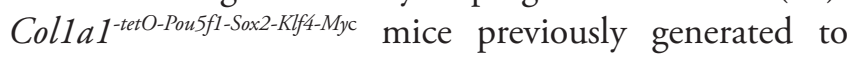
express $4 \mathrm{~F}$ from a tetracycline inducible promoter (17) were crossed with the K14-rtTA mice (14) and the progeny were induced with doxycycline in anagen (PD27) and late catagen (PD44) (Figures 1A and 3A). We were unable to assess time points later than 9 days, because the induced mice became sick and died.

After the induction, we isolated single cell suspensions from the skin and employed fluorescence-activated cell sorting (FACS) using cell surface markers: 

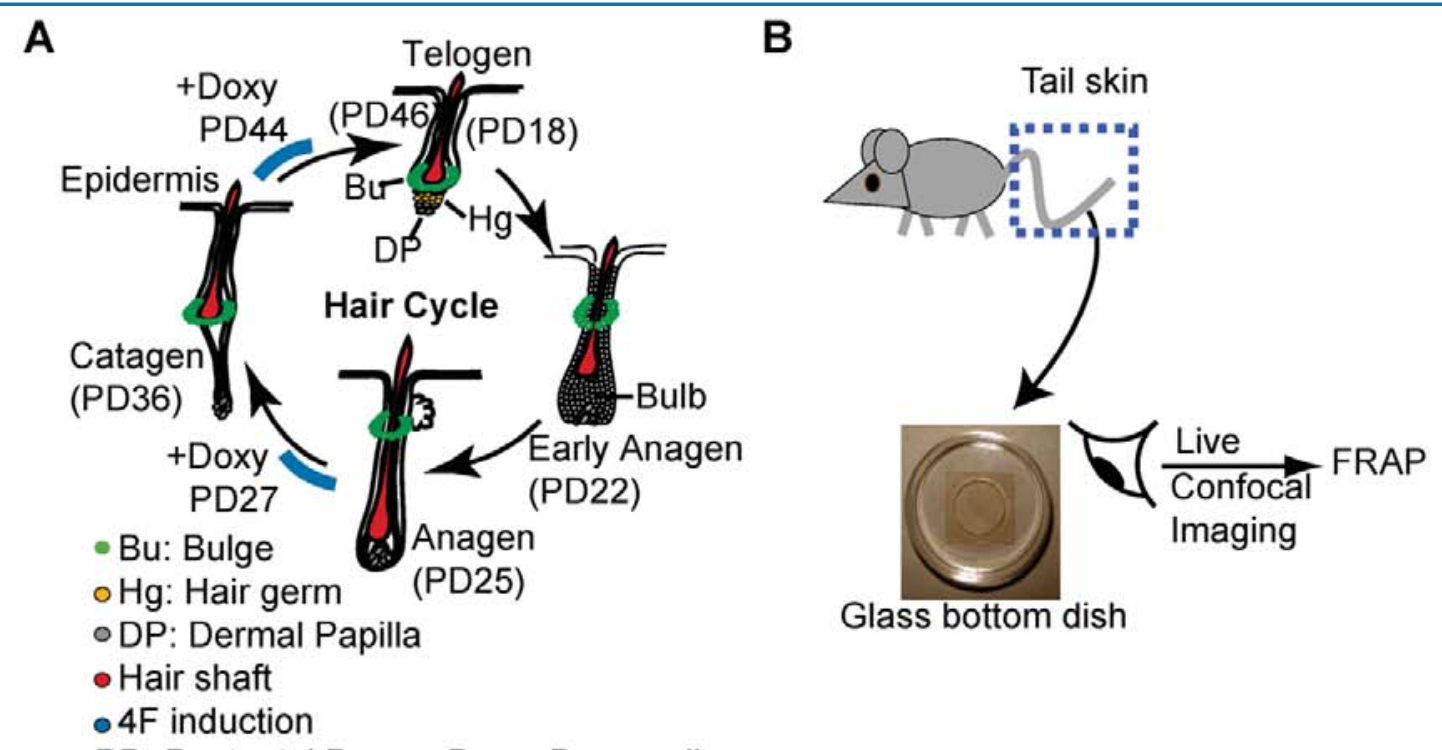

PD: Postnatal Days Doxy: Doxycycline
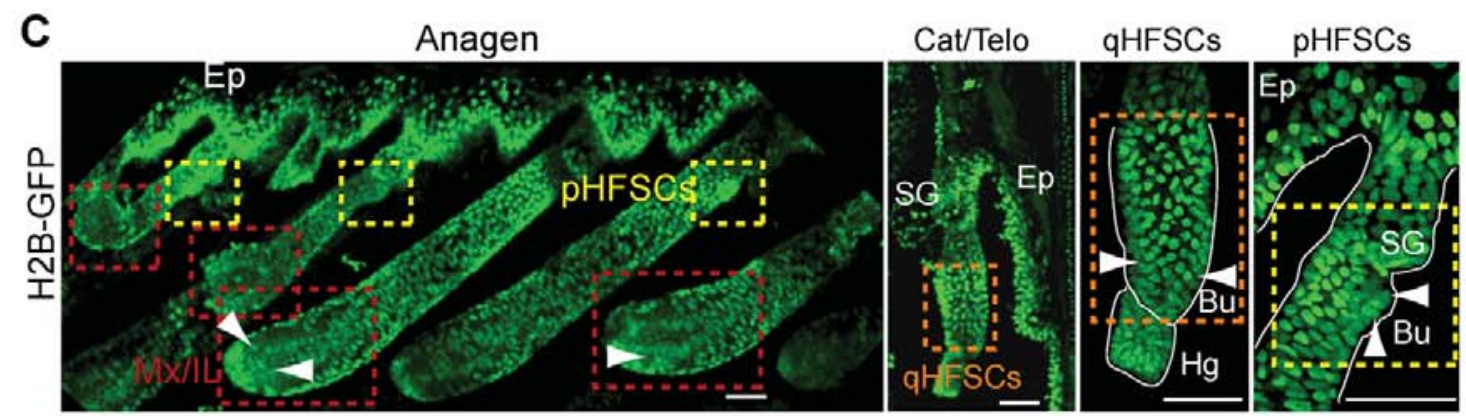

D
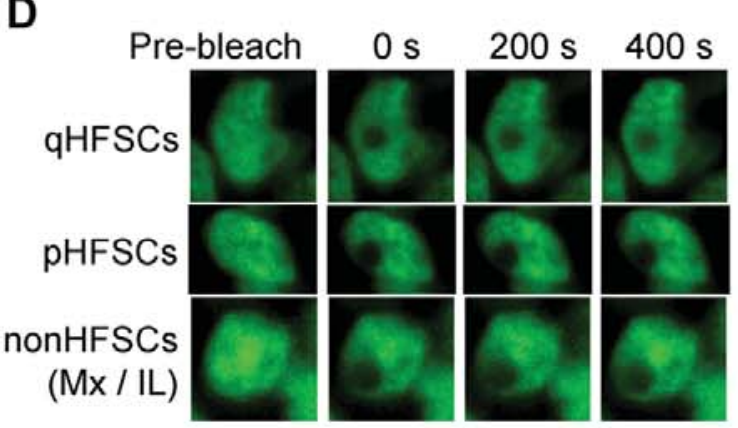

E

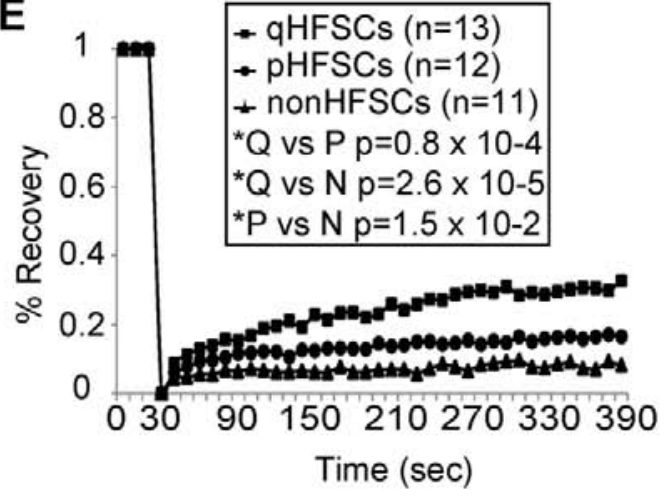

$\mathbf{F}$

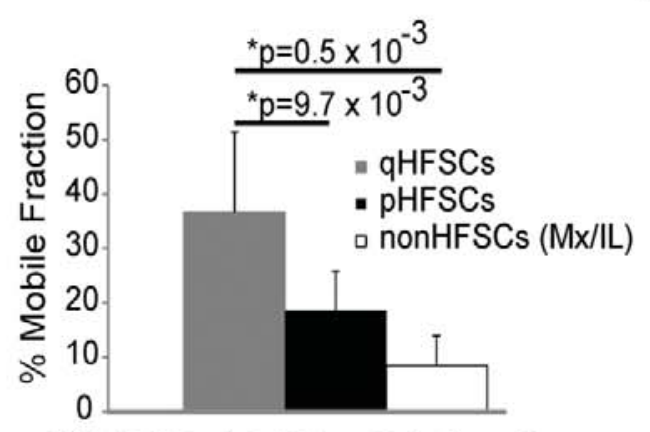

H2B-GFP hair follicle cells in live skin

Fig. 1. - Quiescent HFSCs display high H2B-GFP exchange rates in vivo. (A) Description of hair follicle structure and its morphology during the hair cycle. (B) Confocal live imaging of H2B-GFP expressing mouse skin explant. (C) Representative $\mathrm{z}$-stacks of live imaged skin. Arrow heads indicate FRAP-exposed cells in relevant hair follicle regions, as indicated by dotted box. Scale bars $=50 \mu \mathrm{m}$. (D) Examples of cells that were photobleached are shown. (E) FRAP recovery curves show higher recovery rate in qHFSCs. $\mathrm{n}=$ number of cells. (F) Estimated H2B-GFP mobile fraction in each condition after exponential curve fitting of data from (E). Mx, matrix; IL, Inner layer; q or Q, quiescent; p or P, proliferative; HFSC, hair follicle stem cell. 


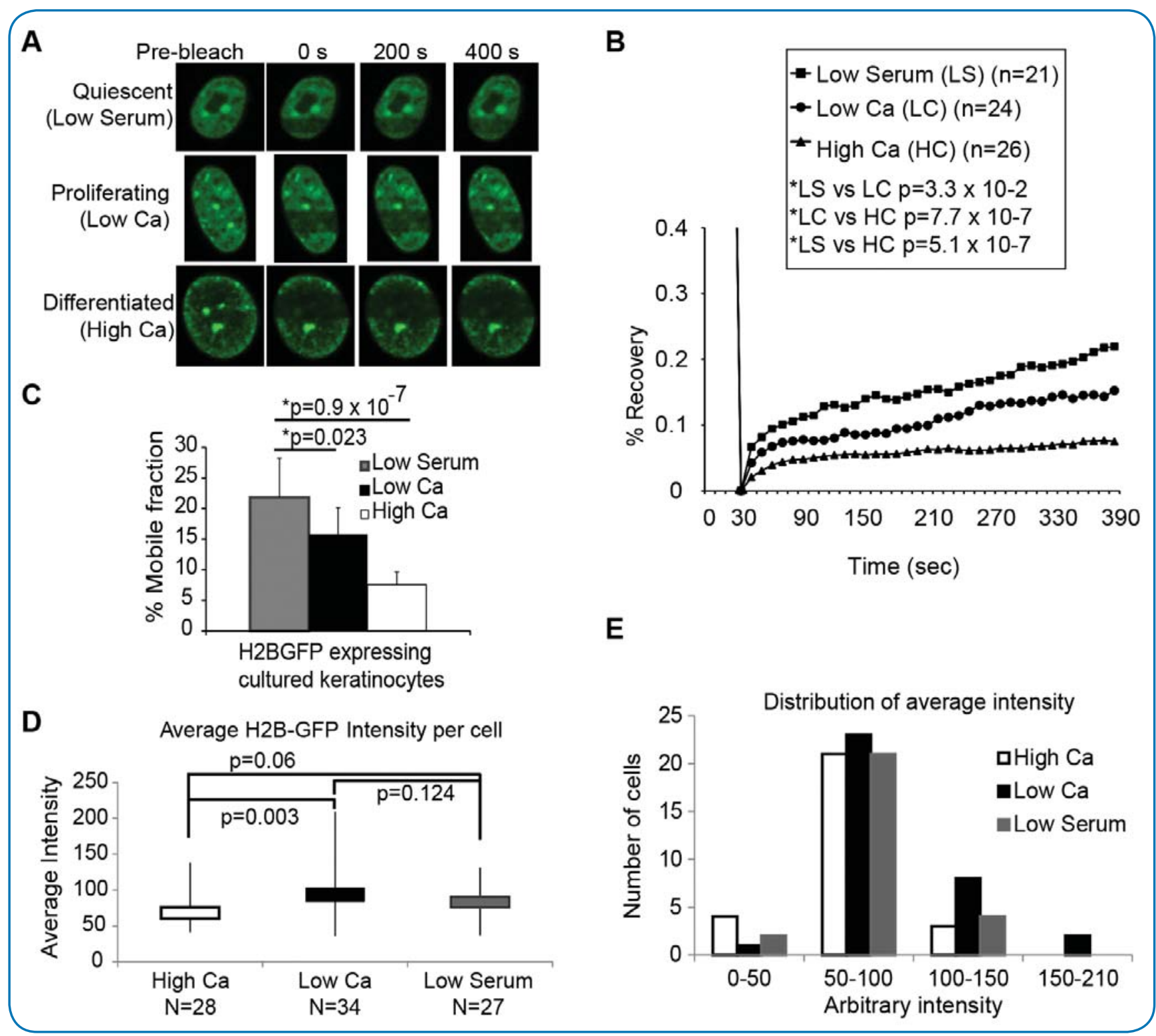

Fig. 2. - Quiescent keratinocytes under in vitro conditions show high H2B-GFP exchange rates.

(A) Examples of H2B-GFP-expressing cells cultured in different conditions that were photobleached are shown at different time points of recovery. (B) FRAP recovery curves show higher recovery rate in keratinocytes that were grown in low serum conditions to induce quiescence. (C) Estimated mobile fraction in each condition after exponential curve fitting from (B). (D) Box-whisker plot of average H2B-GFP intensity per cell under different culture conditions. Average H2B-GFP intensity was measured by drawing a region around each cell and quantifying the average intensity, taking into account the cell shape, total area, and total intensity. (E) Distribution of cells in (D) as a function of H2B-GFP fluorescence. Note that the differences in H2B-GFP intensity are not significant in quiescent (low serum) vs proliferative cells (low $\mathrm{Ca}^{2+}$ ). Although differentiating conditions (high $\mathrm{Ca}^{2+}$ ) show slightly higher $\mathrm{H} 2 \mathrm{~B}-\mathrm{GFP}$ levels they in fact display the lowest recovery rates.

CD34+/a6-integrin+ to isolate the HFSCs and CD34-/a6-integrin+ for non-HFSCs (Fig. 3B). We then extracted RNA to assess the extent of de-differentiation by quantitative reverse-transcriptase PCR (qRT-PCR) of specific markers. Indeed, both proliferative and quiescent HFSCs expressed high levels of $4 \mathrm{~F}$, with variability across different populations (Fig. 3C). To account for this variability the expression level of subsequent markers was first normalized to the control (Gapdh) to calculate the relative fold change, which was further normalized to the level of $4 \mathrm{~F}$ expression in each sample. As expected, we found that quiescent HFSCs significantly down-regulated multiple epithelial skin lineage markers (multiple Keratins, Cd34, a6-integrin) compared to proliferative HFSCs and non-HFSCs, suggesting that these cells were more readily de-differentiated (Fig. 3D). Of note, we do not yet fully understand the correlation between the mRNA and protein level of 4F, as well as the inverse linear relationship between the $4 \mathrm{~F}$ level and lineage markers. However, these results suggest a plausible and testable model that quiescent HFSCs are more readily de-differentiated than 
A

pTRE-Oct4/KIf4/Sox2/Myc $\times$ K14rtTA mice

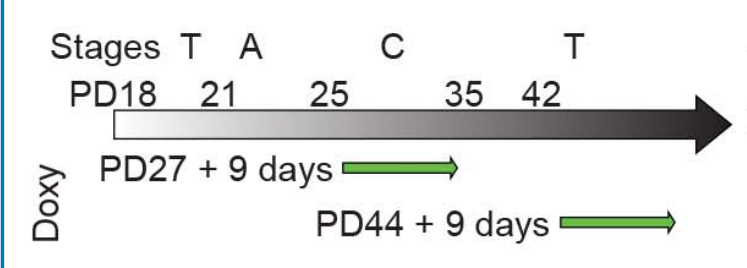

B

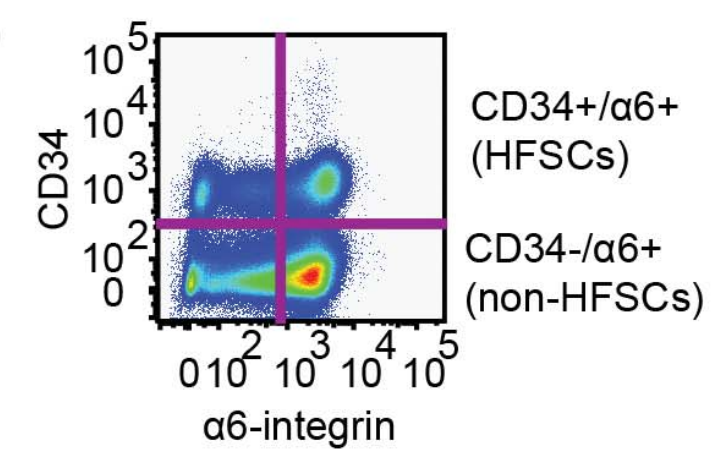

C

T: Telogen

A: Anagen

C: Catagen

PD: Postnatal Days

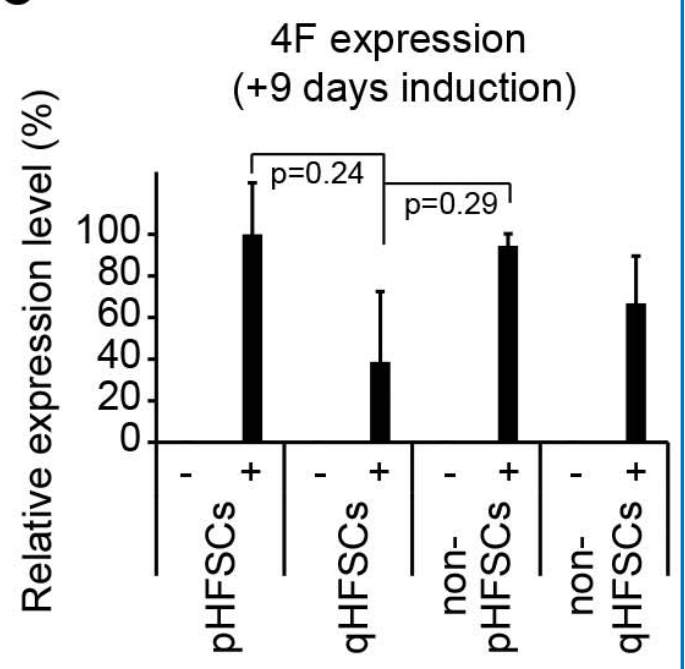

D

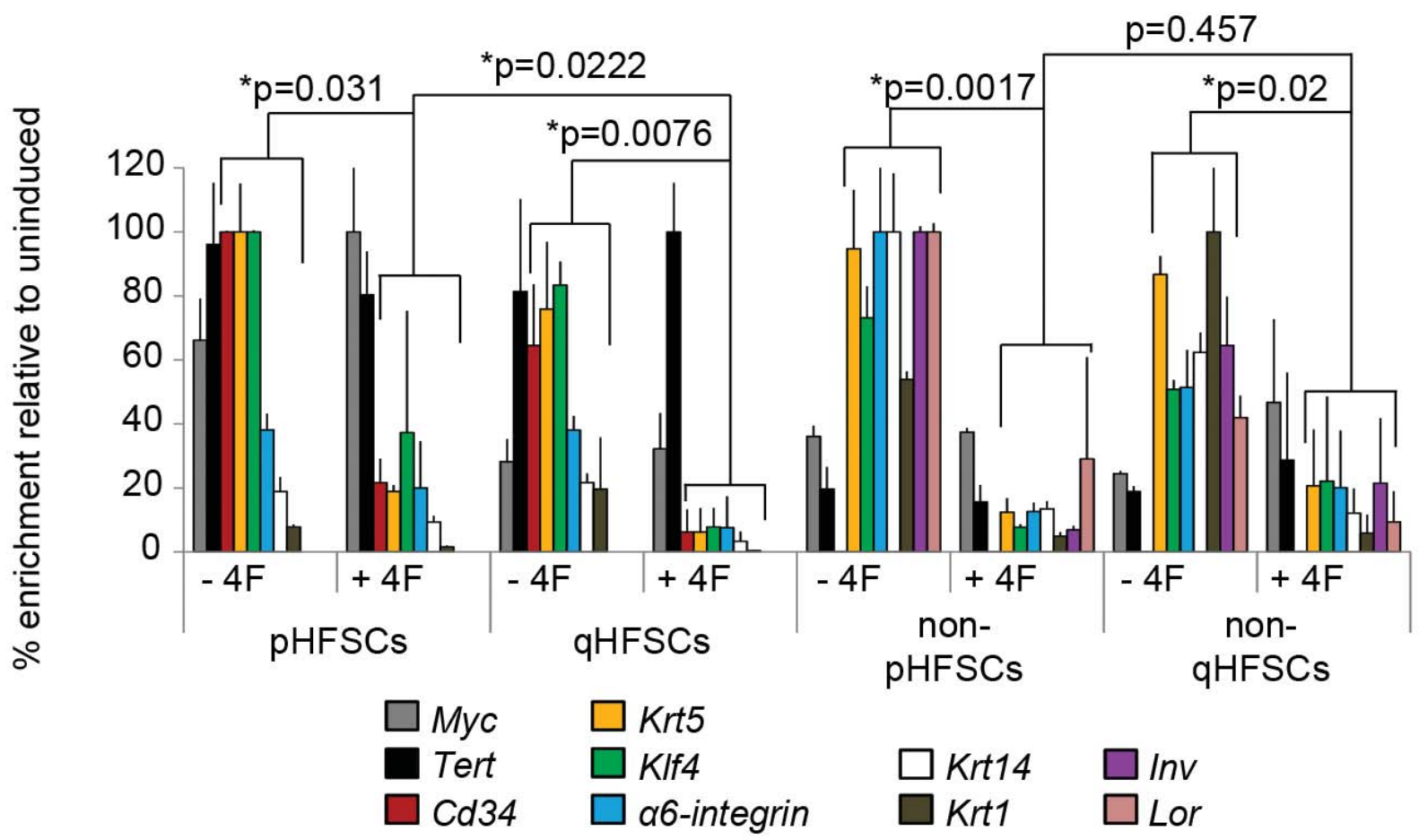

Fig. 3. - Quiescent HFSCs display increased extent of de-differentiation upon induction of Yamanaka factors. (A) Hair cycle and doxycycline induction scheme for $4 \mathrm{~F}$ expression. Tet-inducible mice (17) expressing Yamanaka factors (4F) in response to doxycycline were induced for 9 days at anagen (PD27) and catagen (PD44) stages. (B) Dot plot FACS diagram of skin cells show gating of HFSCs and non-HFSCs using CD34 and a6-integrin for cell isolation. (C) qRT-PCR analysis of relative mRNA expression level of reprogramming factors (Oct4, Klf4, Sox2, and $c M y c=O K S M)$ in FACS-isolated HFSCs and non-HFSCs in anagen ( $\mathrm{p}=$ proliferative) and catagen ( $\mathrm{q}=$ quiescent) from mice treated with doxycycline for 9 days. OKSM are transcribed as a single polycistronic transgenic mRNA $^{17}$ (D) Sorted \pm CD34/a6+ skin cells were subjected to qRT-PCR for mRNAs of skin lineage-genes, normalized to Gapdh and to the level of induced $4 \mathrm{~F}$ at each stage. Normalized signal shows greater reduction in qHFSCs (catagen/telogen) relative to pHFSCs (anagen; $\mathrm{n}=2$ mice/stage). 
proliferative HFSCs and non-HFSCs, which is line with increased plasticity of cells in this state.

In conclusion, we tested plasticity-associated states previously defined in cultured ESCs (1) in mouse skin within the HFSC niche. We show that quiescent HFSCs possess enhanced plasticity-associated states, when compared with proliferative HFSCs and with more differentiated epithelial skin compartments. These results are recapitulated in cultured keratinocytes that were induced in quiescence. In addition, quiescent HFSCs seem to be more readily de-differentiated, suggesting that these cells may bear higher plasticity than that of proliferative HFSCs. Furthermore, our previous work showed that quiescent HFSCs actively maintain globally low histone methylation status, and inhibition of demethylating enzymes perturbed the subsequent activation (9). Taken together, these data suggest a model where the chromatin of quiescent HFSCs is actively maintained in a more dynamic state that conforms with higher plasticity. In the future, assessing how perturbation of such specialized chromatin state (e.g., histone methylation, dynamics of chromatin protein) of HFSCs cause defects in stem cell fate plasticity may unveil a direct mechanistic link between the two.

Our data may have relevance to other tissue stem cell systems and their mechanisms of plasticity of fate acquisition. Some tissue stem cells are preferentially quiescent and/or undergo dynamic stochastic or environmentally driven choices of either differentiation or self-renewal like the hair follicle $(3,4)$. For instance, mouse hematopoietic stem cells reside in G0 quiescence and divide only five times in a lifetime (18) and over-expression of Ezh2 promotes their self-renewal (19), supporting our model. Notably, quiescent muscle stem cells (20) and progenitor $\mathrm{T}$ cells also displayed some globally hypomethylated histones, and this state enhanced reprogramming efficiency in cultured $\mathrm{T}$ cells (11). Perhaps quiescence and plasticity, the most fundamental characteristics of many tissue stem cells, are coupled via homeostatic signals in tissues beyond hair follicles.

\section{MATERIALS AND METHODS}

\section{Mice}

All mice were treated according to Cornell University Institutional Animal Care and Use Committee protocols (IACUC). To obtain transgenic mice expressing Yamanaka factors (15), we crossed K14-rtTA (from E. Fuchs, Rockefeller University, New York, NY) (14) with Colla 1 ${ }^{\text {-tetO-Pouff-Soxz-Kff-Myc }}$ (Jackson Laboratories B6; 129S4-Colla1tm 1(tetO-Pouff-Kff,-Sox2,-Myc) HochlJ, \#011001) (17). Mice with both transgenes were fed with doxycycline chow (Bio-Serv, Flemington, NJ) for 9 days to induce Yamanaka factors.
Fluorescence Recovery After Photobleaching (FRAP)

For invivoFRAP, tail explants of K5-tTAx $p$ TRE-H2BGFP

$(13,21)$ mice were imaged in PBS within 30 minutes from surgery. For in vitro FRAP, keratinocytes from K14-rtTA (14) x $p$ TRE-H2BGFP (13) mice were cultured on MatTek dishes and treated with doxycycline $(2 \mu \mathrm{g} / \mathrm{mL})$ for at least 48 hours to induce H2B-GFP expression. Cells cultured in low $\mathrm{Ca}^{2+}$ media $0.05 \mathrm{mM}$ were proliferating cells. Treatment with high $\mathrm{Ca}^{2+} 2 \mathrm{mM}$ for 48 hours led to keratinocyte differentiation. To keep cells in quiescent state, cells were starved by serial media dilution over 4-5 days. H2B-GFP-expressing keratinocytes were subjected to live-cell imaging at $37^{\circ} \mathrm{C}$. A Zeiss confocal LSM 710, $30 \mathrm{~mW}$ Argon/Neon laser at $80 \%$ power and 10 iterations were used to bleach $1 / 4-1 / 3$ of the nucleus, including both heterochromatin and euchromatin. Images were collected at $1 \%$ power, every 10 seconds for 40 cycles, and processed with MetaMorph imaging software. Data analysis and exponential curve fitting were performed by easy FRAP software (22). All statistical analyses were performed by two-sided student $\mathrm{t}$-test. For FRAP recovery curves, a logarithmic trend line was fit for each curve of individual cells, and the slopes of the curves, representing how fast the recovery was, were subjected to the two-sided student t-test.

\section{Fluorescence Activated Cell Sorting (FACS)}

For all FACS experiments, we used a protocol that has previously been described (21).

\section{Quantitative real-time reverse transcriptase PCR (qRT-PCR)}

A previously described method was used for RNA extraction and qRT-PCR (21). To calculate the reprograming efficiency, we first used a standard $\Delta \Delta \mathrm{CT}$ method to measure the relative fold enrichment of target over control (Gapdh) across different populations. The relative expression of transgenic Oct4/Klf4 and Sox2/Myc were measured using two separate primers (see below for sequence), and the values were averaged to calculate $4 \mathrm{~F}$ expression in each sample. Following, the relative expression value of targets was normalized to the average $4 \mathrm{~F}$ expression in each sample. Primers used for qRT-PCR are listed below: Oct4/Klf4 (transgenic), TCTGTTCCCGTCACTGCTC IAGAGAGTTCCTCACGCCAAC;

Sox2/Myc (transgenic),

ACATGGGTAGTGGGCAATGT /

CGCAGATGAAATAGGGCTGT;

Cd34, AAGGCTGGGTGAAGACCCTTA /

TGAATGGCCGTTTCTGGAAGT;

Krt1, AACCCGGACCCAAAACTTAG /

CCGTGACTGGTCACTCTTCA;

Krt5, CCTGCAGAAGGCCAAGCA /

TGGTGTTCATGAGCTCCTGGTA; 
Krt14, AAGGTCATGGATGTGCACGAT /

CAGCATGTAGCAGCTTTAGTTCTTG; a6-Integrin, TGCAGAGGGCGAACAGAAC /

GCACACGTCACCACTTTGC;

Klf4 (endogenous), ATGGTCAAGTTCCCAGCAAG / TTTGTAAGTCCGGGCATGTT; $c M y c$ (endogenous), TTGAGGAAACGACGAGAACA / GCCAAGGTTGTGAGGTTAGG; Involucrin, CACAATGCCAGGTCTTCACTGA / AGGGTTTGGCCGCTTCTC; Loricrin, TCACTCATCTTCCCTGGTGCTT / GTCTTTCCACAACCCACAGGA; Gapdh, ACTGCCACCCAGAAGACTGT / GATGCAGGGATGATGTTCTG

Author Contributions: JL and TT performed experiments, analyzed the data, designed the project and wrote the paper.

\section{REFERENCES}

1. Meshorer E, et al., Hyperdynamic plasticity in pluripotent embryonic of chromatin proteins stem cells,

Dev. Cell, 22, 233-234 (2006).

2. Mattout A, Meshorer E, Chromatin plasticity and genome organization in pluripotent embryonic stem cells, Current Opinion in Cell Biology, 22, 334-341 (2010).

3. Simons BD, Clevers H, Strategies for homeostatic stem cell self-renewal in adult tissues, Cell, 145, 851-862 (2011).

4. Sada A, Tumbar T, New Insights into Mechanisms of Stem Cell Daughter Fate Determination in Regenerative Tissues, Int. Rev. Cell Mol. Biol., 300, 1-50 (2013).

5. Zhang YV, Cheong J, Ciapurin N, McDermitt DJ, Tumbar T, Distinct Self-Renewal and Differentiation Phases in the Niche of Infrequently Dividing Hair Follicle Stem Cells, Cell Stem Cell, 5, 267-278 (2009).

6. Rompolas P, et al., Live imaging of stem cell and progeny behaviour in physiological hair-follicle regeneration,

Nature, 487, 496-499 (2012).

7. Greco V et al., A Two-Step Mechanism for Stem Cell Activation during Hair Regeneration, Cell Stem Cell, 4, 155-169 (2009).

8. Lee S, et al., High Runx1 levels promote a reversible, more-differentiated cell state in hair-follicle stem cells during quiescence, Cell Rep., 6, 499-513 (2014).

9. Lee J, et al., Signalling couples hair follicle stem cell quiescence with reduced histone $H 3 \mathrm{~K} 4 / \mathrm{K} 9 / \mathrm{K} 27 \mathrm{me} 3$ for proper tissue homeostasis, Nat. Commun., 7, 1-15 (2016).

10. Hawkins RD, et al., Distinct epigenomic landscapes of pluripotent and lineage-committed human cells, Cell Stem Cell, 6, 479-491 (2010).

11. Baxter J, et al. Histone hypomethylation is an indicator of epigenetic plasticity in quiescent lymphocytes, $\mathbf{E M B O}$ J., 23, 4462-4472 (2004).
Acknowledgments: We thank L.N. Tu and D.J. McDermitt for assistance with performing experiments and mouse care, L.G. Sayam for FACS, all personnel in Cornell University CARE for mouse care. Confocal images were acquired through Cornell University Biotechnology Resource Center Imaging Facility. Cytometry core is supported in part by the ESSCF, NYS-DOH, contract \#C026718. Opinions expressed are solely of the author; they do not necessarily reflect the view of ESSCB, the NYS-DOH, or NYS. This work was supported by NIH 2RO1AR053201 and 2R56AR053201 to T.T and T32GM007273 to J.L.
12. Kobielak K, Stokes N, de la Cruz J, Polak L, Fuchs E, Loss of a quiescent niche but not follicle stem cells in the absence of bone morphogenetic protein signaling,

Proc. Natl. Acad. Sci., 104, 10063-10068 (2007).

13. Tumbar T, Defining the Epithelial Stem Cell Niche in Skin, Science, 303, 359-363 (2004).

14. Nguyen H, Rendl M, Fuchs E, Tcf3 Governs Stem Cell Features and Represses Cell Fate Determination in Skin, Cell, 127, 171-183 (2006).

15. Takahashi K, Yamanaka S, Induction of Pluripotent Stem Cells from Mouse Embryonic and Adult Fibroblast Cultures by Defined Factors, Cell, 126, 663-676 (2006).

16. Stadtfeld M, Hochedlinger K, Induced pluripotency: History, mechanisms, and applications, Genes and Development, 24, 2239-2263 (2010).

17. Stadtfeld M, Maherali N, Borkent M, Hochedlinger K, $A$ reprogrammable mouse strain from gene-targeted embryonic stem cells, Nat. Methods, 7, 53-55 (2010).

18. Fuchs E, The Tortoise and the Hair: Slow-Cycling Cells in the Stem Cell Race, Cell, 137, 811-819 (2009).

19. Herrera-Merchan A, et al., Ectopic expression of the histone methyltransferase Ezh2 in haematopoietic stem cells causes myeloproliferative disease, Nat. Commun., 3, 623 (2012).

20. Liu L, et al., Chromatin Modifications as Determinants of Muscle Stem Cell Quiescence and Chronological Aging, Cell Rep., 4, 189-204 (2013).

21. Lee J, et al. Runx1 and 21 synergistically limit the extent of hair follicle stem cell quiescence in vivo,

Proc. Natl. Acad. Sci. U.S.A., 110, 4634-4639 (2013).

22. Rapsomaniki MA, et al., EasyFRAP: An interactive, easy-to-use tool for qualitative and quantitative analysis of FRAP data, Bioinformatics, 28, 1800-1801 (2012). 